\title{
FREGE, POINCARÉ, CARNAP, KRIPKE: CUATRO RÉPLICAS A UN DOGMA KANTIANO Emilio Méndez Pinto*
}

RESUMEN: Presento las réplicas que Gottlob Frege, Henri Poincaré, Rudolf Carnap y Saul Kripke hicieron a la asunción de que aprioridad y necesidad son sinónimos intercambiables, asunción que tomo, junto con las asunciones de que existe una escisión entre las verdades analíticas y las verdades sintéticas y de que existe una dicotomía entre nuestros esquemas conceptuales y el contenido empírico, como un dogma kantiano.

PALABRAS ClAVE: Epistemología, Kant, metafísica.

\section{FREGE, POINCARÉ, CARNAP, KRIPKE: FOUR REPLIES TO A KANTIAN DOGMA}

ABSTRACT: I present the replies that Gottlob Frege, Henri Poincaré, Rudolf Carnap, and Saul Kripke made to the assumption that apriority and necessity are interchangeable synonyms, an assumption that I take, together with the assumptions that there is a split between analytic truths and synthetic truths and that there is a dichotomy between our conceptual schemes and empirical content, as a Kantian dogma.

KEYWORDS: Epistemology, Kant, metaphysics. 


\author{
FREGE, POINCARÉ, CARNAP, \\ KRIPKE: CUATRO RÉPLICAS \\ A UN DOGMA KANTIANO
}

\title{
Introducción
}

$\mathrm{E}_{\text {n este trabajo }}{ }^{1}$ se exponen las réplicas que Frege, Poincaré, Carnap, y Kripke dieron a lo que aquí consideraré como un dogma kantiano: la tesis de que aprioridad y necesidad son sinónimos intercambiables. ${ }^{2}$ En la

${ }^{1}$ Aquí desarrollo más detalladamente algunas de la tesis que expuse en "Sobre proposiciones $a$ priori y contingentes y a posteriori y necesarias considerando teoremas geométricos elementales", Estudios 114 (2015): 175-182, y "Necesidad matemática y mundos posibles", Estudios 130 (2019): 7-25. En cuanto a los "dogmas del empirismo" (la tríada clásica que consiste en los dos dogmas señalados por Quine y el señalado por Davidson), sobre los que escribo aquí, escribí también algo al respecto de uno de ellos en "Una paradoja en la máxima quineana de que ningún enunciado es inmune a la revisión", Estudios 120 (2017): 127-133.

${ }^{2}$ Esta relación de sinonimia se debe a Kripke. Véase: Saul Kripke, El nombrar y la necesidad, primera parte expondré esta tesis kantiana, así como sus orígenes y las razones para considerarla un dogma filosófico. En la segunda, tercera, cuarta y quinta partes expondré las réplicas fregeana, poincariana, carnapiana y kripkeana.

Las motivaciones de cada una de estas réplicas son distintas, así como también sus respectivas estrategias. Conviene señalar esto tan solo sea para decir que este trabajo no intenta proponer alguna suerte de criterio replicador unificado. Por la misma razón, no ofrezco conclusiones al respecto, más

trad. por Margarita Valdés (México: UNAM, Instituto de Investigaciones Filosóficas, 2005), 156-157. 
allá de las que puedan irse entretejiendo a lo largo de la lectura. Por último, conviene señalar tales motivaciones y estrategias, aunque se harán explícitas a lo largo de este trabajo, pero antes es necesario un comentario general sobre la relevancia de exponer las cuatro réplicas a la vez.

Creo que es interesante advertir tanto la naturaleza como las motivaciones que, ante una misma idea (nuestro dogma kantiano), tuvieron cuatro réplicas pertenecientes a cuatro tradiciones intelectuales distintas (aunque no necesariamente adversas en todos sus detalles): el logicismo, el intuicionismo, el empirismo lógico $y$, a falta de un mejor término, una variante de la epistemología modal. ${ }^{3}$ En cuanto

${ }^{3}$ Para tres variantes del logicismo, véase: Agustín Rayo, "Logicism reconsidered", en The Oxford handbook of philosophy of mathematics and logic, ed. por Stewart Shapiro (Oxford: Oxford University Press, 2005), 203-235. Para las distintas motivaciones entre el logicismo de Frege y el de Russell, véase: Alejandro Tomasini, Los atomismos lógicos de Russell y Wittgenstein (México: UNAM, Instituto de Investigaciones Filosóficas, 2012), 127-128. Para la distinción entre intuicionismo, matemáticas intuicionistas y finitismo, véase: Alexander George y Daniel Velleman, Philosophies of mathematics (Delaware: Blackwell, 2002), 89-172. Para la exposición del intuicionismo oficial (en otras palabras, la versión brouweriana, no la versión poincariana), véanse: Carl Posy, "Intuitionism and philosophy", y D.C. McCarty, "Intuitionism in mathematics", ambos en The Oxford handbook of philosophy of mathematics and logic, 318-355 y 356-386. Para una muestra de la influencia de Carnap en el empirismo lógico, véase: The Cambridge companion to logical empiricism, ed. por Alan Richardson y Thomas Uebel (Cambridge: Cambridge University Press, 2007), 374-376. Por último, para el antecedente inmediato de la epis- tal, este trabajo no tiene pretensiones que vayan mucho más allá de la simple exposición, y esto obedece, en buena medida, a la propia naturaleza de nuestro tema, sin que esto signifique que no sea posible rebasar el marco de una mera exposición, a la manera de una síntesis general o de conclusiones particulares.

En el caso de Frege, su motivación estuvo restringida, al menos en su proyecto logicista original, ${ }^{4}$ al caso de las proposiciones aritméticas, pero nunca al caso de las proposiciones geométricas, sobre las que siempre fue un kantiano: "Al llamar sintéticas a prio$r i$ a las verdades de la geometría, Kant descubrió su verdadera esencia". ${ }^{5}$ Frege, pues, siguiendo a Kant, consideraba que las verdades geométricas eran verdades sintéticas a priori. ${ }^{6}$ Pero

temología modal de Kripke que consideramos a lo largo de este trabajo, véase: Saul Kripke, Identidad y necesidad, trad. por Margarita Valdés (México: UNAM, Instituto de Investigaciones Filosóficas, 1978); para su continuación, como la llama el propio Kripke, véase: Saul Kripke, Reference and Existence (Oxford: Oxford University Press, 2018).

${ }^{4}$ Gottlob Frege, "Sources of knowledge of mathematics and the mathematical natural sciences", en Gottlob Frege: Posthumous writings, ed. por Hans Hermes, Friedrich Kambartel y Friedrich Kaulbach (Chicago: University of Chicago Press, 1979), 267-274.

${ }^{5}$ Jesús Mosterín, Los lógicos (Madrid: Austral, 2007), 108.

${ }^{6}$ Empero, Frege, a diferencia de Kant, distinguió entre juzgar y pensar, y es por ello que, para él, lo que hay son verdades matemáticas (ya sean geométricas o aritméticas), y no meramente, como en Kant, juicios matemáticos (ya sean geométricos o aritméticos). Por otra parte, el marcado kantismo 
no siguió a Kant en cuanto a la naturaleza de las verdades aritméticas, que para Frege no eran sintéticas y a prio$r i$, como en Kant, sino analíticas y $a$ priori. Su carácter analítico se debe, según Frege, a que cuando se lleva a cabo la prueba de una proposición aritmética hasta sus últimas consecuencias, se llega a leyes y definiciones lógicas generales. Su carácter $a$ priori se debe a que su prueba puede derivarse de leyes generales que no necesitan $o$ no admiten ninguna prueba empírica. Como argumentaré más adelante, la diferencia fundamental entre la concepción kantiana de lo $a$ priori y la concepción fregeana de lo a priori es que la primera, además de tener un carácter epistemológico, tiene también un carácter metafísico, mientras que la segunda solo tiene un carácter epistemológico.

El caso de Poincaré es diametralmente opuesto al de Frege: si este fue un kantiano con respecto a la naturaleza de la geometría, pero no de la aritmética, Poincaré fue un kantiano con respecto a la naturaleza de la aritmética, pero no de la geometría (el caso de Brouwer fue exactamente igual al de Poincaré, aunque por mo-

de Frege con respecto a la naturaleza de las proposiciones geométricas lo llevó a ningunear, por supuestamente absurdas, las proposiciones fundamentales de las geometrías no euclidianas. Su kantismo también explica en buena medida la controversia que Frege sostuvo con Hilbert sobre los fundamentos de la geometría. tivaciones filosóficas distintas). Sin embargo, contrariamente a lo que este hecho podría sugerir, la réplica poincariana al dogma kantiano según el cual aprioridad y necesidad son sinónimos intercambiables no solo es pertinente en la geometría, sino también en la aritmética. Esto, porque, como veremos, la intuición matemática poincariana es relevantemente distinta de la intuición matemática kantiana, tanto en su aspecto geométrico como en su aspecto aritmético.

El caso de Carnap es fundamentalmente distinto de los dos anteriores porque no tiene ningún sesgo kantiano en absoluto. ${ }^{7}$ Así, a diferencia de Kant y de Frege, para quienes las verdades geométricas tienen un carácter sintético y a priori, para Carnap las verdades geométricas tienen un carácter sintético solo si se trata de verdades geométricas aplicadas, pero entonces no tienen un carácter $a$ priori; las verdades geométricas tienen un carácter a priori solo si se trata de verdades geométricas puras, pero entonces no

${ }^{7}$ Podemos encontrar en Bolzano los orígenes de una crítica de la filosofía (matemática) kantiana que no tuvo ningún sesgo kantiano. Bernard Bolzano, Contribuciones a una presentación mejor fundamentada de las matemáticas, trad. por Emilio Méndez Pinto (México: Biblioteca Digital del ILCE, Unesco). Para un recuento del antikantismo de Bolzano, así como de su influencia en la filosofía (analítica), véase: Mark Textor, “Bolzano's anti-Kantianism: From a priori cognitions to conceptual truths", en The Oxford handbook of the history of analytic philosophy, ed. por Michael Beaney (Oxford: Oxford University Press, 2015), 227-249. 
tienen un carácter sintético. Por el otro lado, a diferencia de Kant y de Poincaré (y de Brouwer), para Carnap los juicios aritméticos no son sintéticos y a priori, sino analíticos y a priori. Esto, porque nuestro conocimiento matemático, si bien tiene un carácter apriorístico, está basado en nuestras convenciones para emplear símbolos lógicos y matemáticos. ${ }^{8}$

Por último, en el caso de Kripke, su réplica al dogma kantiano según el cual aprioridad y necesidad son sinónimos intercambiables consiste en señalar que "necesidad epistemológica" y "necesidad metafísica" no son sinónimos intercambiables. ${ }^{9}$ Así, hay proposiciones a priori contingente-

${ }^{8}$ Esta solución convencionalista, sumamente simplificada aquí, fue la que adoptó el empirismo lógico para “acomodar” las verdades matemáticas y lógicas en la concepción empirista del mundo. $\mathrm{Al}$ respecto, véase: Michael Kremer, "The whole meaning of a book of nonsense: Reading Wittgenstein's Tractatus", en The Oxford handbook of the history of analytic philosophy, 451-485. Para más sobre esta solución y sobre las célebres críticas de Quine al respecto, véase: Michael Resnik, "Quine and the web of belief', en The Oxford handbook of philosophy of mathematics and logic, 412-436.

${ }^{9}$ Tal como utilizaremos estos términos a lo largo de este trabajo, un conocimiento epistemológicamente necesario es un conocimiento a priori, un conocimiento no sujeto a ninguna contingencia fáctica (creo que así es justamente como Kant entiende lo a priori), mientras que algo metafísicamente necesario es algo metafísicamente verdadero en todos los mundos posibles. En concordancia con el contenido de este trabajo, los "mundos posibles" los entenderemos a la manera kripkeana, y no (por ejemplo) a la manera lewisiana. Para la "manera kripkeana", véase: Kripke, El nombrar y la necesidad, 20-25. Para la "manera lewisiana", véase: David mente verdaderas y proposiciones $a$ posteriori necesariamente verdaderas.

\section{Aprioridad y necesidad como sinónimos intercambiables}

\author{
En la adenda a El nombrar y la nece- \\ sidad, Kripke escribió:
}

Tal vez debo mencionar también que no pude encontrar en Kant la caracterización de una verdad a priori como una verdad que puede conocerse independientemente de la experiencia; hasta donde puedo ver, Kant se refiere solo al conocimiento $a$ priori de enunciados particulares, lo cual no supone la modalidad adicional. [...] Y, por supuesto, cuando Kant usa "necesario" para un tipo de proposición y "a priori" para un modo de conocimiento, no se le puede culpar de caer en la práctica común contemporánea de tratar a los dos términos como sinónimos intercambiables. Resulta claro, desde las primeras páginas de la Crítica [de la razón pura], que la tesis de que el conocimiento de que algo es necesario tiene que ser a priori es considerada por Kant como una tesis importante y sustantiva, aunque obvia. ${ }^{10}$

Lewis, On the plurality of worlds (Selangor: Blackwell, 2013).

${ }^{10}$ Kripke, El nombrary la necesidad, 156-157. Me parece que lo que Kripke está diciendo en las primeras líneas de este pasaje es que no pudo encontrar, en la Crítica de la razón pura, ningún contraejemplo a su propia tesis, esto es, a la tesis de 
Que Kant haya considerado obvia la tesis de que el conocimiento de que algo es necesario tiene que ser a prio$r i$ es lo que la hace un dogma. En su momento, Kant también consideró como obvia la tesis de que hay una escisión entre las verdades analíticas y las sintéticas y la tesis de que hay una dicotomía entre nuestros esquemas conceptuales y el contenido empírico. Por los trabajos de Quine y de Davidson al respecto, estas dos tesis "obvias" acabaron formando parte de la tríada de "dogmas del empirismo"." Kripke dice que a Kant "no se le puede culpar de caer en la práctica común contemporánea de tratar a los dos términos [aprioridad y necesidad] como sinónimos intercambiables". Esta apología puede igualmente extenderse al primero de sus otros dos

que, para Kant, el conocimiento de que algo es necesario tiene que ser a priori. De aquí, creo, que Kripke haga énfasis en que no encontró en Kant "la caracterización de una verdad a priori como una verdad que puede conocerse independientemente de la experiencia", porque, de haberla encontrado, ello habría supuesto un contraejemplo a su propia lectura de Kant. Por su parte, Kant explicita, en las líneas introductorias a su Crítica de la razón pura, lo que entiende con conocimiento a priori (un conocimiento que, si bien comienza con la experiencia, no tiene sus fuentes en la experiencia) y lo que entiende con conocimiento a posteriori (un conocimiento que, al igual que el a priori, no solo comienza con la experiencia, sino que, también, tiene sus fuentes en ella). Immanuel Kant, Critique of pure reason, trad. por J. M. D. Meiklejohn (Londres: The Colonial Press, 1900), 1.

${ }^{11}$ El tercer dogma, también señalado por Quine, es que nuestro conocimiento tenga una relación directa con nuestra experiencia sensible. dogmas, que, como señala Quine, "fue presagiado por la distinción de Hume entre relaciones de ideas y cuestiones de hecho, y en la distinción de Leibniz entre verdades de razón y verdades de hecho". ${ }^{12}$ Con respecto al dogma señalado por Davidson, la apología de Kant trasciende su contemporaneidad, porque su dogma es común a "filósofos de muchas corrientes [que] son propensos a hablar de esquemas conceptuales. Los esquemas conceptuales, se nos dice, son modos de organizar la experiencia; son sistemas de categorías que dan forma a los datos de la sensación [...] Incluso aquellos pensadores que están seguros de que hay solo un esquema conceptual están en el dominio del concepto de los esquemas; incluso los monoteístas tienen religión". ${ }^{13}$ Según esta caracterización, Kant sería uno de los monoteístas.

El dogma kantiano que nos concierne, a saber, el que sostiene que "aprioridad" y "necesidad" son sinónimos intercambiables, también fue presagiado por Leibniz, para quien, si algo es necesariamente verdadero, entonces se debe a un conocimiento $a$ priori, mientras que, si algo es contin-

${ }^{12}$ W. V. O. Quine, From a logical point of view: Nine logico-philosophical essays (Cambridge: Harvard University Press, 1980), 20.

${ }^{13}$ Donald Davidson, "On the very idea of a conceptual scheme", Proceedings and addresses of the American Philosophical Association 47 (1973-1974): 5-20. 
gentemente verdadero, se debe a un conocimiento a posteriori. En términos leibnizianos: si algo es tal que su contrario es imposible, entonces se debe a una verdad de razón, mientras que, si algo es tal que su contrario es posible, entonces se debe a una verdad de hecho.

Para Kant, lo a priori no solo concierne a la epistemología (esto es, a nuestro modo de conocer), sino también a la metafísica (esto es, al modo en el que, por la naturaleza de nuestra razón, debemos conocer). En este punto, su postura se desmarca no solo de la de Leibniz, sino también de la de cualquier realista trascendental. ${ }^{14}$ Para los realistas trascendentales, cualquier instancia del principium tertii exclusi es a priori decidible; para los empiristas, es decidible solo a posteriori, y para el idealismo trascendental es a priori indecidible.

Kant llevó hasta sus últimas consecuencias el dogma leibniziano según el cual si algo es necesario, entonces obedece a una verdad a priori, mientras que si algo es contingente, se debe a una verdad a posteriori. Lo llevó hasta sus últimas consecuencias por-

${ }^{14}$ Tomo de Carl Posy el término "realista trascendental" para referirme a cualquier metafísico clásico anterior a Kant. Posy lo distingue del "idealista trascendental", que sería Kant, principalmente en que, para este último, las antinomias de la razón pura - por ejemplo, si el mundo físico ocupado es espacialmente finito o infinito- no son disyunciones lógicas, como sí lo son para el realista trascendental. Posy, "Intuitionism and philosophy", 348. que sustituyó una relación de causalidad entre estos conceptos epistemológicos y metafísicos por una relación de sinonimia: en Kant, si $x$ se conoce apriorísticamente, entonces $x$ es ipso facto necesariamente verdadero. ${ }^{15}$ Aquí, $\mathrm{x}=$ cualquier proposición de la geometría euclidiana; $\mathrm{x}=$ cualquier proposición de la mecánica newtoniana. Paralelamente, las proposiciones de la geometría euclidiana y de la mecánica newtoniana son sintéticas, lo que significa que, no obstante su carácter apriorístico (tanto en términos epistemológicos como modales o metafísicos), nos dan información sobre el mundo (o, en otros términos, en tales enunciados los predicados no están contenidos en sus sujetos). ${ }^{16}$

Digo "en otros términos" porque Kant entendía la dicotomía analítico / sintético en términos de la relación sujeto-predicado (relación que se remonta a los silogismos aristotélicos).

${ }^{15}$ Esto se debe a que, para la doctrina kantiana, ni nuestras intuiciones sensibles a priori (el espacio y el tiempo) ni nuestras categorías pueden ser contradichas por la experiencia sensible. Kant, Critique of pure reason, III-XI.

${ }^{16} \mathrm{En}$ el caso de la mecánica newtoniana, el aspecto sintético de sus proposiciones es poco controvertible, porque el mismo Newton sostuvo que "la geometría está basada en la práctica mecánica". Isaac Newton, Principios matemáticos de la filosofia natural, trad. por Antonio Escohotado (Madrid: Tecnos, 2011), 6. Para una defensa de los enunciados sintéticos definidos como aquellos cuyos predicados no están contenidos en sus sujetos, véase: Hao Wang, From mathematics to philosophy (Nueva York: Routledge, 2016), 251-279. 
Es por esto que un juicio analítico - aquel en el que el concepto del predicado ya es parte del concepto del sujeto- no dice nada sobre el mundo, no nos da información sobre el mundo; en pocas palabras, no transmite información fáctica, mientras que, por el otro lado, con un juicio sintético sucede exactamente lo contrario. No es ningún accidente histórico el que posturas manifiestamente antikantianas relativas a la naturaleza de las matemáticas hayan puesto énfasis en el carácter analítico de las proposiciones matemáticas. ${ }^{17}$

Kant, al momento de haber no solo establecido, sino fundamentado la posibilidad de juicios sintéticos $a$ priori, fracturó un dogma de su época, que el conocimiento a priori es, $a$ fortiori, analítico. Y de ninguna manera es trivial el significado de esta empresa para el proyecto crítico kantiano, sino todo lo contrario, según lo dicho por el propio Kant en la Introducción a la Crítica de la razón pura y según, también, la lectura de Strawson al respecto:

A lo largo de la Crítica [de la razón pura] Kant hace uso frecuente de cierta distinción $[\ldots]$ : la distinción

${ }^{17}$ Dos ejemplos paradigmáticos son Carl Hempel, Sobre la naturaleza de la verdad matemática, trad. por Emilio Méndez Pinto (México: Biblioteca Digital del ILCE, Unesco), 5-6, y Hans Reichenbach, Objetivos y métodos del conocimiento fisico, trad. por Eugenio Ímaz (México: FCE, 1996), 41-42. entre las proposiciones analíticas y sintéticas a priori. Se dice que ambos tipos de proposiciones tienen en común que pueden ser conocidas no solo como verdaderas sino de tal forma que ninguna experiencia podría desconfirmarlas ["infirmarlas", en el original] o presentar un contraejemplo. En este sentido, ambos tipos de proposiciones se contrastan con proposiciones empíricas verdaderas, que son aquellas que podemos saber que son verdaderas solo porque se ven confirmadas por la experiencia, y en la medida en que esto sucede. Kant mantiene que mientras que el carácter a priori de las proposiciones analíticas no plantea ningún problema filosófico profundo, algo distinto sucede con las proposiciones sintéticas a priori. De hecho, dice en la Introducción que todo el problema a cuya solución está dedicada la Crítica de la razón pura puede resumirse en la siguiente pregunta concisa: ¿cómo son posibles los juicios sintéticos a priori $?^{18}$

Empero, en su sistema filosófico, Kant ${ }^{19}$ instituyó (cayó en la prác-

${ }^{18}$ Peter F. Strawson, Los límites del sentido: Ensayo sobre la Crítica de la razón pura de Kant, trad. por Carlos Thiebaut Luis-André (México: UNAM, Instituto de Investigaciones Filosóficas, 2019), 44-45. Para la referencia directa a Kant: $\mathrm{Cri}$ tique of pure reason, 1-18.

${ }^{19}$ Para un argumento a favor de la idea de Kant como el constructor de un sistema filosófico, véase: Margaret D. Wilson, "Substance and system: Perplexities of the geometrical order", The Harvard Review of Philosophy II, núm. 1 (1992): 8-13. 
tica común de su época, siguiendo a Kripke) el dogma según el cual aprioridad y necesidad son sinónimos intercambiables.

\section{La réplica de Frege}

En Los fundamentos de la aritmética, Frege se atuvo a tres principios: 1) siempre separar claramente lo psicológico de lo lógico, lo subjetivo de lo objetivo; 2) nunca preguntar por el significado de una palabra aislada, sino únicamente en el contexto de una proposición; 3) nunca perder de vista la distinción entre concepto y objeto. ${ }^{20}$ Para los propósitos de este ensayo, nos interesa el primer principio, porque es aquel que sugiere prescindir del psicologismo (kantiano, por ejemplo) al momento de dar cuenta de la naturaleza de los objetos de la aritmética. ${ }^{21}$ Con respecto a las

${ }^{20}$ Gottlob Frege, Los fundamentos de la aritmética, trad. por Emilio Méndez Pinto (México: Biblioteca Digital del ILCE, Unesco), 9.

${ }^{21}$ No obstante, Russell achacó psicologismo al propio Frege. Bertrand Russell, Las doctrinas lógicas y aritméticas de Frege, trad. por Emilio Méndez Pinto (México: Biblioteca Digital del ILCE, UNESCO). Por otra parte, en la bibliografía filosófica sobre estos asuntos no es inusual encontrar la afirmación de que Frege achacó psicologismo a John Stuart Mill, y que, por tanto, Frege objetó la filosofía matemática de Mill por psicologista. Esto es falso. Frege objetó la filosofía matemática de Mill por empirista, y fue Husserl, no Frege, quien achacó psicologismo a la filosofía matemática de Mill. díadas analítico / sintético y a priori / a posteriori, Frege escribe que

resultan cuatro posibles combinaciones, de las cuales una, analítico a posteriori, puede ser eliminada. ${ }^{22}$ Aquellos que, junto con Mill, han decidido a favor de lo a posteriori no tienen una segunda opción, ${ }^{23}$ así que solo nos quedan dos posibilidades por examinar, a saber, sintético $a$ priori y analítico. Kant se decide por la primera. En ese caso, no hay otra alternativa sino la de invocar una intuición pura como el fundamento último de nuestro conocimiento de tales juicios, aunque es difícil decir si esta es espacial o temporal, o cualquier otra cosa. ${ }^{24}$

Linnebo compendia lo anterior como sigue (poniendo de su propia cosecha) ${ }^{25}$

\begin{tabular}{c|cc} 
& analítico & sintético \\
\hline a priori & Frege & $\begin{array}{c}\text { Platón (precopernicano), } \\
\text { Kant, Brouwer } \\
\text { aposteriori }\end{array}$ \\
& $\mathrm{x}$ & $\begin{array}{c}\text { (copernicano) } \\
\text { Mill, Quine }\end{array}$
\end{tabular}

${ }^{22}$ Puede ser eliminada porque, por definición, no puede haber juicios analíticos a posteriori.

${ }^{23} \mathrm{Al}$ ser imposibles los juicios analíticos $a$ posteriori, y dado que Mill se decidió a favor de lo a posteriori, solo le queda la opción de los juicios sintéticos a posteriori. Dada esta restricción, a Quine también le quedó solamente la opción de los juicios sintéticos a posteriori.

${ }^{24}$ Frege, Los fundamentos de la aritmética, 23.

${ }^{25}$ Øystein Linnebo, Philosophy of mathematics (Princeton: Princeton University Press, 2017), 19. 
Frege, pues, para el caso de la aritmética, no objetó lo a priori en lo "sintético a priori" de Kant, sino lo sintético (para el caso de la geometría, no objetó ni lo sintético ni lo a priori kantianos). Objetó lo sintético porque, para Frege, como vimos, si llevamos a cabo la prueba de una proposición aritmética hasta sus últimas consecuencias, llegamos a leyes y definiciones lógicas generales, lo que, entre otras cosas, quiere decir que las proposiciones aritméticas no transmiten información fáctica, información relativa a los hechos del mundo. ${ }^{26}$ Esto último no quiere decir que, para Frege, la aplicación efectiva de las leyes de la aritmética sea algo trivial para la universalidad de las leyes de la aritmética, sino más bien que, para Frege (a diferencia, por ejemplo, de Descartes), dicha aplicación es normativa, en el mismo sentido en el que la lógica fue normativa para C. S. Peirce, Ramsey y Aristóteles.

Pero en un sentido no trivial, Frege objetó lo a priori en su connotación kantiana; y no solo eso: se propuso precisar qué quiso decir realmente Kant cuando empleó este término. Al examinar la distinción entre lo a priori / a posteriori y lo analítico / sintético en las primeras páginas de Los

${ }^{26}$ Para esta postura (logicista), véase: Hempel, Sobre la naturaleza de la verdad matemática y Frank P. Ramsey, Los fundamentos de las matemáticas, trad. por Emilio Méndez Pinto (México: Biblioteca Digital del ILCE, Unesco). fundamentos de la aritmética, Frege escribió en una nota a pie de página: "Es claro que [...] no pretendo asignar un nuevo sentido a estos términos, sino solo establecer precisamente qué quisieron decir con ellos algunos autores, particularmente Kant". ${ }^{27}$

¿Qué objetó Frege a lo a priori kantiano? Objetó su carácter trascendentalista: que, en sus intentos por resolver el problema filosófico ${ }^{28}$ de los objetos matemáticos, Kant haya recurrido a la naturaleza de la mente humana; en términos fregeanos, al psicologismo (que Frege identificó con el idealismo trascendental de Kant, en tanto que este, si bien no resuelve el problema ontológico de los números, sí resuelve el problema de la nosensitividad de los conceptos-número). Sobre esta estrategia kantiana, por así llamarla, escribe Frege:

El enfoque histórico, con su objetivo de detectar cómo comienzan las cosas y de llegar desde estos orígenes a un conocimiento de su naturaleza, es perfectamente legítimo; pero también tiene sus límites. Si todo estuviese en un flujo continuo y nada se mantuviese fijo para todo el tiempo, no

${ }^{27}$ Frege, Los fundamentos de la aritmética, 12.

${ }^{28}$ Problema filosófico en el sentido extenso del término, que atañe a cuestiones ontológicas, metafísicas y epistemológicas. Para una erudita e iluminativa exposición del problema de los objetos matemáticos en la historia de la filosofía, véase: Michael Detlefsen, "Formalism", en The Oxford handbook of philosophy of mathematics and logic, 236-317. 
habría ninguna posibilidad de llegar a conocer algo sobre el mundo y todo estaría inmerso en confusión. Parecería que damos por supuesto que los conceptos brotan en la mente individual como las hojas de un árbol, y creemos descubrir su naturaleza al estudiar su nacimiento: buscamos definirlos psicológicamente en términos de la naturaleza de la mente humana. Pero esta consideración hace todo subjetivo, y si la seguimos hasta el final elimina la verdad. ${ }^{29}$

Me gustaría argumentar que Frege no objeta el aspecto epistemológico de lo a priori $^{30}$ kantiano, sino su ingrediente metafísico. En otras palabras, objetó que la distinción a priori / a posteriori derivara del contenido de los juicios (distinción / concepción kantiana) y no de la justificación de los juicios (distinción / concepción fregeana). ${ }^{31}$

Para Kant, al no haber una distinción entre "necesidad epistemológica"

${ }^{29}$ Frege, Los fundamentos de la aritmética, 7 .

${ }^{30} \mathrm{Al}$ que positivamente entiende como "una justificación para hacer un juicio. Cuando no hay tal justificación, se desvanece la posibilidad de trazar las distinciones. Un error a priori es, de esta forma, un sinsentido, como lo es, digamos, un concepto azul". Frege, Los fundamentos de la aritmética, 12.

${ }^{31}$ Timothy Williamson ha sostenido en varios lugares que la distinción a priori/a posteriori es epistemológicamente inútil porque oscurece ciertos patrones epistémicos significativos. Aunque no discutiré esto por ser un tanto ajeno a nuestros temas, me parece que sus objeciones a este respecto son pertinentes para la distinción/concepción kantiana de lo a priori/a posteriori, pero no necesariamente para la fregeana. y "necesidad metafísica", si algo es conocido a priori, entonces ipso facto es necesariamente verdadero. En Frege, en cambio, un juicio aritmético es a priori si su prueba puede derivarse de leyes generales que no necesitan o no admiten ninguna prueba empírica. El hecho de que pueda derivarse implica que un juicio a priori (un juicio aritmético elemental, por ejemplo), si bien puede no necesitar prueba empírica alguna para su validación, ello no significa que no admita prueba empírica alguna. Desde luego, como en muchas otras cuestiones filosóficas, el diablo está en los detalles: la implicación anterior es válida si el término disyuntivo "o" en "un juicio es a priori si su prueba puede derivarse de leyes generales que no necesitan $o$ no admiten ninguna prueba empírica" tiene un carácter inclusivo (no necesita o no admite $o$ ambas), pero no es válida si tiene un carácter exclusivo (no necesita o no admite pero no ambas). En mi opinión, la interpretación correcta del uso de este término disyuntivo por parte de Frege es que tiene un carácter inclusivo. ${ }^{32}$ Escribe Frege:

Desde luego, fórmulas numéricas como $7+5=12$ y leyes como la ley

${ }^{32}$ Una disyunción inclusiva verdadera es aquella que sigue siendo verdadera si uno o ambos de sus argumentos son verdaderos, mientras que una disyunción exclusiva verdadera es aquella que sigue siendo verdadera únicamente cuando uno u otro, pero no ambos, de sus argumentos es verdadero. 
asociativa de la adición están tan ampliamente establecidas por las innumerables aplicaciones que se hacen de ellas cada día que casi parecería ridículo intentar traerlas a disputa al demandar su prueba. Pero en la naturaleza de las matemáticas siempre está preferir una prueba, donde sea posible, a cualquier confirmación por inducción. Euclides ofrece pruebas de muchas cosas que cualquiera concedería sin preguntar. $Y$ fue cuando el hombre rechazó estar satisfecho incluso con los estándares de rigor de Euclides que llegó a las indagaciones puestas en marcha por el axioma de las paralelas. ${ }^{33}$

El axioma de las paralelas de Euclides parecería ser, en las manos de Frege, un contraejemplo de la "implicación" que establecí líneas arriba relativa a que un juicio a priori, si bien puede no necesitar prueba empírica alguna para su validación, ello no significa que no admita una prueba empírica, y que esto último es, no obstante, relevante para su verdad. Pero no lo es el ejemplo aritmético de Frege, porque el enunciado " $7+5=12$ " es a priori en tanto que su prueba, si bien puede derivarse de leyes generales que no necesitan ninguna prueba empírica, no tiene que derivarse de leyes generales que no admitan prueba empírica alguna, y esto último, la aplicación de los conceptos-número, de ningún

\footnotetext{
${ }^{33}$ Frege, Los fundamentos de la aritmética, 11.
}

modo fue algo trivial para Frege. Para el caso geométrico, en cambio (y esto obedece al kantismo de Frege con respecto a la naturaleza de nuestros juicios geométricos), el axioma de las paralelas de Euclides debe derivarse de leyes generales que, si bien podrían llegar a admitir pruebas empíricas, tienen que derivarse, en última instancia, de leyes generales cuya corroboración empírica es irrelevante para los propósitos de su verdad. La historia de la geometría - en las manos de Gauss, Riemann, Bolyai, Lobachevski y un largo etcétera- mostró que esto fue un error.

\section{La réplica de Poincaré}

$\mathrm{Si}$, como dijimos al comienzo, Frege fue un kantiano con respecto a la naturaleza de la geometría pero no de la aritmética, Poincaré fue un kantiano con respecto a la naturaleza de la aritmética pero no de la geometría. Poincaré fue un kantiano con respecto a la naturaleza de los juicios aritméticos porque, en su opinión, los juicios aritméticos son sintéticos $a$ priori en tanto que, conjuntamente, 1) no pueden demostrarse analíticamente; 2) no pueden reducirse a identidad alguna; 3) no pueden establecerse experimentalmente. ${ }^{34}$ Así pues,

${ }^{34}$ Henri Poincaré, Ciencia y método, trad. por Emilio Méndez Pinto (México: Biblioteca Digital del ILCE, Unesco), 93. 
en opinión de Poincaré, los trabajos $\operatorname{logicistas}^{35}$ de su época (aquí Poincaré se remite a los trabajos de Couturat, Russell y Peano, aunque en otros lugares alude también a Whitehead) de ninguna manera resolvieron - a favor de Leibniz - la vieja controversia entre este y Kant con respecto a la existencia de los juicios sintéti$\cos$ a priori.

Por otra parte, Poincaré no fue un kantiano con respecto a la naturaleza de los juicios geométricos porque, en su opinión, los axiomas geométricos no pueden ser intuiciones sintéticas $a$ priori. Si lo fueran, "estarían impuestas sobre nosotros con tal fuerza que no podríamos concebir una posición contraria, ni podríamos construir sobre ella un edificio teórico. No habría, pues, geometría no euclidiana". ${ }^{36} \mathrm{No}$ habría geometría no euclidiana (ni en su versión hiperbólica o lobachevskiana ni en su versión elíptica o riemanniana) porque, si nuestras intuiciones geométricas fuesen a priori en el sentido kantiano, como dice Poincaré, se nos impondrían: no habría juicios geométricos que, siendo a priori

${ }^{35}$ Poincaré no utilizó este término, sencillamente porque en su época no era ni de lejos un término de uso común. Grosso modo, podemos entender el logicismo como la perspectiva que sostiene que las matemáticas son una parte de la lógica, más allá de la vaguedad de esta formulación. Agustín Rayo, "Logicism reconsidered", 203-235.

${ }^{36}$ Henri Poincaré, Ciencia e hipótesis, trad. por Emilio Méndez Pinto (México: Biblioteca Digital del ILCE, Unesco), 36. (en el sentido kantiano del término), no fuesen, a la vez, necesariamente verdaderos. ${ }^{37}$

Ahora bien, si en el caso de los juicios geométricos hay una diferencia relevantemente significativa entre Kant y Poincaré con respecto a su carácter apriorístico, ¿la hay también en el caso de los juicios aritméticos, a pesar del kantismo de Poincaré a este respecto? Sostengo que sí. Creo que, a pesar de la simpatía de Poincaré por la filosofía de la aritmética de Kant, su "intuicionismo matemático" no es estrictamente kantiano. ${ }^{38} \mathrm{La}$ intuición matemática poincariana se asemeja, en mi opinión, a la intuición matemática de Turing, ${ }^{39}$ que concierne a ideas intuitivas sin ninguna identificación particular. Parsons distinguió entre la intuición matemática gödeliana (que tiene por modelo la noción husserliana de intuición) y la intuición matemática kantiana (que concierne a una forma de sensibilidad). ${ }^{40}$ En mi opinión, la

${ }^{37}$ Para un examen del convencionalismo geométrico en general y el convencionalismo geométrico poincariano en particular, véase: Rudolf Carnap, Una introducción a la filosofía de la ciencia, trad. por Emilio Méndez Pinto (México: Biblioteca Digital del ILCE, Unesco).

${ }^{38}$ Habida cuenta, por ejemplo, de que "no es verdad, como argumentó Kant, que la intuición es un medio puro a priori de conocimiento. [...] Más bien es fuerza del hábito enraizada en inercia psicológica". Hans Hahn, Empiricism, logic and mathematics (Londres: D. Reidel, 1980), 93, 101.

${ }^{39}$ Alan Turing, Systems of logic based on ordinals (Princeton: Seeley G. Mudd Manuscript Library, 2014).

${ }^{40}$ Charles Parsons, Mathematics in philosophy. Selected essays (Nueva York: Cornell University Press, 2005), 24. 
intuición matemática poincariana no cae ni bajo la noción husserliana (o gödeliana), que tiene un marcado componente intelectual, ni bajo la intuición matemática kantiana, que tiene un marcado componente metafísico. En cambio, la intuición matemática poincariana tiene un carácter marcadamente psicológico. El "intuicionismo matemático" de Poincaré es relevantemente distinto de los intuicionismos matemáticos de Brouwer, Heyting, Weyl, Kolmogorov, Gentzen, Kleene y Dummett, por ejemplo, en tanto que estos, a diferencia del intuicionismo matemático de Poincaré, tienen un marcado carácter lógicofilosófico. ${ }^{41}$

${ }^{41}$ Si bien tanto Brouwer como Poincaré eran partidarios del kantismo para explicar la naturaleza de los juicios aritméticos, los motivos de uno y otro eran distintos. Esto puede elucidarse, me parece, atendiendo a las respectivas razones que tuvieron para no adherirse al kantismo al momento de dar cuenta de la naturaleza de los juicios geométricos: mientras que Brouwer "señala que las geometrías no euclidianas muestran que no hay ningún conjunto uniforme de enunciados a priori acerca de la naturaleza del espacio, similar a lo que puede decirse acerca de la naturaleza del tiempo, [y por tanto] acepta la perspectiva de Kant acerca del tiempo como una intuición a priori, rechaza la pretensión paralela de Kant de que el espacio, también, es una forma de intuición. Los sistemas de coordenadas de los números reales serán representantes de la geometría, y así, dice Brouwer, no necesitamos apelar a una intuición espacial independiente" (Posy, "Intuitionism and philosophy", 347, nota 66), Poincaré, por su parte, aunque también debido a la naturaleza de las geometrías no euclidianas, afirmó que los enunciados geométricos tenían un carácter esencialmente convencional. Pero esto fue porque Brouwer encontró, en los mismos sistemas de coor-
Sobre la relación entre el descubrimiento matemático (desde luego, en un sentido no platónico del término) y nuestra psicología, Poincaré dice:

La génesis del descubrimiento matemático es un problema que debe inspirar a los psicólogos con el más vivo interés, porque este es el proceso por el cual la mente humana parece tomar menos prestado del mundo exterior, y en donde actúa, o por lo menos así lo parece, solo por sí misma y sobre sí misma, de tal suerte que al estudiar el proceso del pensamiento geométrico podríamos esperar llegar a lo que resulta más

denadas de los números reales como "representantes" de la geometría, la perspectiva kantiana del tiempo como una intuición a priori, y por eso rechazó, al menos según la lectura de Posy, una intuición a priori extra, la relativa al espacio. Pero ese no fue, ni remotamente, el motivo de Poincaré para no adoptar el kantismo al momento de dar cuenta de la naturaleza de los juicios geométricos: fue justamente porque no encontró ningún paralelismo kantiano (en el sentido que atribuye Posy a Brouwer) en nuestros razonamientos geométricos que Poincaré rechazó la doctrina kantiana acerca de la naturaleza de la geometría. En segundo lugar, pero relacionado con el punto anterior, las formulaciones de Brouwer acerca de los defectos de las matemáticas clásicas - defectos cuyas causas identifica, en buena medida, en el incesante uso del principio del tercero excluido- son formulaciones dirigidas a los fundamentos de las matemáticas, mientras que las formulaciones "intuicionistas" de Poincaré están más bien dirigidas al quehacer matemático, no a supuestos defectos ni a sus (supuestos) fundamentos. Para esta lectura de Brouwer, véase: John von Neumann, "The formalist foundations of mathematics", en Philosophy of mathematics, ed. por Paul Benacerraf y Hilary Putnam (Cambridge: Cambridge University Press, 1998), 61. 
esencial en la mente humana. [...] Un primer hecho debe sorprendernos, o mejor dicho debería sorprendernos si no estuviésemos acostumbrados a él. ¿Cómo es que hay personas que no comprenden las matemáticas? Si esta ciencia solo recurre a las reglas de la lógica - aquellas aceptadas por toda mente bien formada- - si su evidencia está fundada sobre principios comunes a todos los hombres, y que nadie excepto un loco se atrevería a negar, ¿cómo es que hay tantas personas completamente impermeables a ella? No hay nada misterioso en el hecho de que no todo mundo es capaz de descubrir. Que una persona sea incapaz de retener una demostración que alguna vez ha aprendido es todavía comprensible. Pero lo que parece más sorprendente, cuando lo consideramos, es que alguien sea incapaz de comprender un argumento matemático en el momento mismo en el que se le muestra. Y, sin embargo, aquellos que solo pueden seguir el argumento con dificultad son mayoría; esto es incontestable, y la experiencia de los maestros de educación secundaria ciertamente no me va a contradecir. Y aún más, ¿cómo es posible el error en las matemáticas? Un intelecto sano no debería cometer ningún error lógico, y sin embargo hay mentes muy agudas que no darán un paso en falso en un argumento pequeño tal como los que debemos hacer en las acciones ordinarias de la vida, pero que son incapaces de seguir o repetir, sin error, las demostraciones matemáticas que sin duda son más largas, pero que son, después de todo, solamente acumulaciones de pequeños argumentos exactamente análogos a aquellos que en principio resultan tan fáciles. ¿Es necesario añadir que los mismos matemáticos no son infalibles? ${ }^{42}$

Si antes dije que el intuicionismo matemático de Poincaré se diferencia de los intuicionismos matemáticos de Brouwer, Heyting, etc., porque estos, a diferencia de aquel, tienen un marcado carácter lógico-filosófico, sostengo ahora, siguiendo lo que dice Poincaré en el párrafo anterior, que el intuicionismo matemático de Poincaré se diferencia del intuicionismo matemático de Kant en que el intuicionismo kantiano, a diferencia del poincariano, es infalible. Es infalible porque, para Kant, si las proposiciones de la geometría euclidiana tienen un carácter sintético a priori, se requiere el componente de nuestro conocimiento relativo a nuestra facultad de conocer (nuestro conocimiento a priori) "para dar cuenta del carácter necesario y por lo tanto a priori de nuestro conocimiento del espacio tal como se materializa en la geometría. Dicho conocimiento no deriva de la experiencia, y sin embargo, nos dice cómo debe ser el espacio". ${ }^{43}$ En

${ }^{42}$ Henri Poincaré, Ciencia y método, 28.

${ }^{43}$ Barry Stroud, The significance of philosophical skepticism (Oxford: Oxford University Press, 1984), 149. 
pocas palabras, nuestra experiencia sensible no solo no puede contradecir a nuestra intuición sensible $a$ priori (en este caso, la relativa al espacio), sino que tampoco puede engañarla. Empero, habida cuenta de las geometrías no euclidianas, nuestra intuición sensible a priori (en el sentido kantiano) se ve tanto contradicha como engañada por nuestra experiencia sensible.

Poincaré se pregunta: ¿cómo es posible el error en las matemáticas? Más decididamente: ¿por qué los mismos matemáticos no son infalibles? Una parte de la respuesta tiene que ver con que, en una demostración matemática larga, cometer errores lógicos no es algo inusual en "intelectos sanos" o en "mentes muy agudas". La otra parte tiene que ver justamente con nuestra tesis: lo a priori poincariano, si bien presente en nuestros juicios aritméticos y determinante para la creación matemática, ${ }^{44}$ no es, a diferencia de Kant, metafísicamente determinante, en el sentido de que (por ejemplo) nuestros sentidos no puedan contradecir a nuestras intuiciones sensibles a priori (y, si lo hacen, entonces habría que dudar de nuestros sentidos, mas no de nuestras intuiciones sensibles a priori, que, para Kant, son infalibles).

${ }^{44}$ Tanto así que, para Poincaré, la lógica (contraparte de la intuición en el proceso de la creación matemática), si bien segura, es muy poco expeditiva, $y$, al obligarnos a ir paso a paso, lo que nos da son andaderas, y no alas.

\section{La réplica de Carnap}

De entre las cuatro réplicas que aquí consideramos al dogma kantiano según el cual aprioridad y necesidad son sinónimos intercambiables, sin duda la réplica de Carnap es la más decidida. Lo es porque, como dije al comienzo, la réplica carnapiana no tiene ningún sesgo kantiano: Frege lo tuvo, porque siempre fue un kantiano respecto de la naturaleza de las proposiciones geométricas; Poincaré lo tuvo, porque fue un kantiano en cuanto a la naturaleza de las proposiciones aritméticas, y Kripke lo tuvo, porque (como veremos), si bien la concepción kripkeana de lo a priori constituye una salida del dogma kantiano según el cual "aprioridad" y "necesidad" son sinónimos intercambiables, en la medida en que admite verdades que son a priori sin tener que serlo, la concepción kripkeana no admite proposiciones matemáticas contingentemente verdaderas, es decir, sean (epistemológicamente) a priori o a posteriori, sino que son (metafísicamente) necesariamente verdaderas, conclusión con la que Kant concordaría.

Ahora bien, en el caso de Carnap, su réplica ${ }^{45}$ a la filosofía (matemática) kantiana tuvo lugar en dos frentes,

${ }^{45}$ Réplica que, en general, seguirían los proponentes del empirismo lógico. El caso paradigmático fue el de Alfred J. Ayer. 
aunque ambos dirigidos a desmontar la creencia kantiana según la cual todos los juicios matemáticos son juicios sintéticos a priori: 1) la réplica a la creencia kantiana de que los juicios aritméticos son juicios sintéticos a priori, y 2) la réplica a la creencia kantiana de que los juicios geométricos son juicios sintéticos a priori. La réplica 1) es, per se, interesante, pero es irrelevante para los propósitos de este trabajo, porque Carnap (y los que le antecedieron y siguieron en este sentido) objetó esencialmente el aspecto "sintético" de los juicios sintéticos a priori de Kant relativos a la aritmética. ${ }^{46} \mathrm{Es}$, pues, la réplica 2) la que nos interesa, porque con ella Carnap quiebra no solo la relación (kantiana) indisoluble entre el carácter sintético y el carácter a priori de los juicios geométricos, sino también la relación (nuevamente kantiana) entre aprioridad y necesidad.

Carnap quebró la relación positiva entre el carácter sintético y el carácter a priori de los juicios geométricos al establecer una diferencia fundamental con respecto a la naturaleza empírica de los juicios geométricos: si estos son a priori (es decir, son significativamente independientes de la experiencia sensible), entonces no son sintéticos (es decir, no nos dan información sobre el mundo); si, por el

${ }^{46}$ También objetarían lo a priori kantiano con respecto a los juicios aritméticos, pero, como dije, por razones ajenas a los propósitos de este trabajo. contrario, los juicios geométricos son sintéticos (es decir, nos dan información sobre el mundo), entonces no son a priori (es decir, no son significativamente independientes de la experiencia sensible). En términos de Einstein, ${ }^{47}$ lo anterior significa que las proposiciones de la geometría pura son certeras, pero no acerca de la realidad, mientras que las proposiciones de la geometría aplicada son acerca de la realidad, pero no certeras.

Por otra parte, Carnap quebró la relación sinonímica kantiana entre aprioridad y necesidad al haber mostrado la existencia de juicios geométricos que, si bien pueden ser $a$ priori, no son necesarios. ${ }^{48} \mathrm{~A}$ este respecto, en mis trabajos anteriores he recurrido a la proposición geométrica euclidiana de que "la suma de los ángulos internos de un triángulo es $=180^{\circ}$ "; ahora recurriré, a fin de mostrar lo mismo, a dos ejemplos más tomados también de Carnap. ${ }^{49} \mathrm{Si}$ la

${ }^{47}$ De donde surge esta distinción. Einstein enunció esta distinción en un escrito de 1923 que criticaba un texto que Poincaré escribió 20 años antes, titulado "Experimento y geometría". El escrito de Poincaré se encuentra en Poincaré, Ciencia e hipótesis. El de Einstein en Albert Einstein, Sidelights on relativity (Nueva York: Dutton, 1923).

${ }^{48}$ Más específicamente: juicios geométricos euclidianos que pueden ser a priori en el sentido de que su verdad no requiere una demostración fáctica y que, no obstante, son contingentes, en el sentido de que su contrario es metafísicamente (y lógica, empírica y conceptualmente) posible.

${ }^{49}$ Rudolf Carnap, Una introducción a la filosofia de la ciencia. Carnap recurrió a un cuarto ejemplo a fin de mostrar las respectivas diferencias 
proposición "la suma de los ángulos internos de un triángulo es $=180^{\circ}$ " es contingente a priori, igualmente lo son las proposiciones relativas al número de paralelas ${ }^{50}$ y a la medida de curvatura. ${ }^{51}$ En resumen: ${ }^{52}$

\begin{tabular}{l|c|c|c}
\hline & & $\begin{array}{c}\text { Suma de } \\
\text { los ángulos } \\
\text { internos de }\end{array}$ & \\
geometría & $\begin{array}{c}\text { Número } \\
\text { de paralelas }\end{array}$ & $\begin{array}{c}\text { Medida de } \\
\text { curvángulora }\end{array}$ \\
\hline Lobachevski & $\infty$ & $<180^{\circ}$ & $<0$ \\
\hline Euclides & 1 & $180^{\circ}$ & 0 \\
\hline Riemann & 0 & $>180^{\circ}$ & $>0$ \\
\hline
\end{tabular}

\section{La réplica de Kripke}

Kripke objetó el dogma kantiano según el cual necesidad y aprioridad son sinónimos intercambiables en dos frentes. Por un lado, al haber mostrado cómo puede haber proposiciones $a$ posteriori necesariamente verdaderas $\mathrm{y}$, por el otro, al haber mostrado cómo

entre las geometrías euclidiana, lobachevskiana y riemanniana. Sin embargo, este cuarto ejemplo, relativo a la proporción de la circunferencia de un círculo con respecto a su diámetro - que en la geometría euclidiana es igual a $\pi$, en la hiperbólica es $>\pi$, y en la riemanniana es $<\pi$ - no me parece que sea un ejemplo propicio para nuestros propósitos, porque no creo que el enunciado geométrico (euclidiano) "La proporción de la circunferencia de un círculo con respecto a su diámetro es $=\pi$ " sea una proposición a priori en nuestro sentido, esto es, una proposición cuya verdad sea cognoscible independientemente de la experiencia sensible.

${ }^{50} \mathrm{El}$ quinto postulado de Euclides.

${ }^{51}$ Relativa, por ejemplo, a la superficie de una esfera.

${ }^{52}$ Rudolf Carnap, Una introducción a la filosofia de la ciencia, 132. puede haber proposiciones a priori contingentemente verdaderas. Desde que Kripke y Putnam (el Putnam del realismo externo) argumentaron a favor de dichas posibilidades, surgió toda una bibliografía filosófica a favor o en contra. Pero lo que me interesa mostrar - más allá del contenido de sus propuestas, más allá de una réplica a ellas (como veremos siguiendo a Michael Weisberg), más allá de un recurso para salvar algo de las intuiciones de Kripke-Putnam al respecto (como veremos siguiendo a Agustín Rayo) e incluso más allá de salvar algo de la doctrina kripkeana a expensas de sacrificar su parte más fuerte (como veremos siguiendo a Gareth Evans de la mano de David Papineau) — ${ }^{53}$ es que el hecho de que existan proposiciones a posteriori necesariamente verdaderas y proposiciones a priori contingentemente verdaderas socava los dogmas kantianos según los cuales: 1) los juicios a priori, aun siendo sintéticos, son necesariamente verdaderos, $\mathrm{y}$ 2) los juicios a posteriori, forzosamente sintéticos, son contingentemente verdaderos. ${ }^{54}$

${ }^{53}$ Esto último, porque si bien Evans reconoce que no hay ningún problema con enunciados cognoscibles a priori superficialmente contingentes, entiende que hay problemas serios con enunciados cognoscibles a priori profundamente contingentes.

${ }^{54}$ Para una exposición detallada de las necesidades a posteriori, véase: Stephen Yablo, "Is conceivability a guide to possibility?", Philosophy and Phenomenological Research 53, núm. 1 (1993): 1-42. Para un buen resumen de las clásicas críticas de 
Un ejemplo del primer tipo, el de proposiciones a posteriori necesariamente verdaderas, es "El agua es $\mathrm{H}_{2} \mathrm{O}$ "; un ejemplo del segundo tipo, el de proposiciones a priori contingentemente verdaderas, es "La barra del metro patrón de París mide un metro de largo". La primera proposición es epistemológicamente contingente, pero metafísicamente necesaria: aunque erróneamente, podríamos haber "descubierto" (si nuestra química fuera defectuosa, por ejemplo) que la composición química del agua no es $\mathrm{H}_{2} \mathrm{O}$, sino XYZ (el ejemplo es de Hilary Putnam); por el otro lado, la proposición es metafísicamente necesaria porque es metafísicamente verdadera, en todos los mundos posibles, que el agua es $\mathrm{H}_{2} \mathrm{O}$. La segunda proposición, en cambio, es epistemológicamente necesaria, pero metafísicamente contingente: sabemos, $a$ priori, que "la barra del metro patrón de París mide un metro de largo", pero ello pudo haber sido de otro modo.

Hilary Putnam también recurrió al caso de "El agua es $\mathrm{H}_{2} \mathrm{O}$ " para mostrar

Michael Dummett a Kripke, críticas que tienen el propósito explícito de defender a Frege de Kripke, véase: William C. Smith, "Dummett and rigid designators", Philosophical Studies 37, núm. 1 (1980): 93-103. Por último, para un argumento en contra de la práctica de asemejar la teoría de los tipos naturales de Putnam a la teoría de los tipos naturales de Kripke, práctica que hemos seguido en este trabajo, véase: Ian Hacking, "Putnam's theory of natural kinds and their names is not the same as Kripke's", Principia 11, núm. 1 (2007): 1-24. que hay proposiciones metafísicamente necesarias que, no obstante, son lógica y epistemológicamente contingentes, en el sentido de que no es ni lógica ni epistemológicamente imposible (como ya vimos con Kripke) que el agua sea XYZ (XYZ = composición química del agua), pero sí es metafísicamente imposible. ${ }^{55}$ En opinión de Michael Weisberg, para establecer estos presupuestos, tanto Kripke como Putnam recurren a "la idea de que las clases del lenguaje común y las clases científicas se alinean o se pueden correlacionar entre sí una a una". ${ }^{56}$ Weisberg es crítico de esta correlación. Al respecto, escribe:

Una mirada más atenta al agua muestra que no existe este tipo de correspondencia elemento por elemento entre clases químicas y del lenguaje corriente. De hecho, el uso de términos de clases en química suele ser sensible al contexto, y en casos en que los químicos quieren evitar toda ambigüedad, usan un conjunto muy complejo y matizado de términos de clases, ninguno de los cuales podría asociarse razonablemente con el solo término de clase del lenguaje común "agua". Como no podemos limitarnos

${ }^{55}$ Hilary Putnam, El significado de "significado”, trad. por Jorge Flematti (México: UNAM, Instituto de Investigaciones Filosóficas, 1984).

${ }^{56}$ Michael Weisberg, "El agua no es $\mathrm{H}_{2} \mathrm{O}$ ", en Filosofia de la química: síntesis de una nueva disciplina, coord. por Davis Baird, Eric Scerri y Lee McIntyre (México: FCE, 2011), 500-501. 
a acudir a la química para encontrar una clase química única que pueda usarse para determinar la extensión de "agua", no hay ningún sentido estricto en el que el agua sea $\mathrm{H}_{2} \mathrm{O}$, porque aquello que el agua es exactamente depende del contexto en que se dice "agua". ${ }^{57}$

Quizá podríamos salvar las intuiciones Kripke-Putnam a este respecto recurriendo a Rayo: "Al aceptar 'estar compuesto de agua solo es estar compuesto de $\mathrm{H}_{2} \mathrm{O}$ ', por ejemplo, uno se compromete a la postura de que sería absurdo que haya algo compuesto de agua pero no compuesto de $\mathrm{H}_{2} \mathrm{O}$ ". ${ }^{8}$ Sea como sea, hay más de un ejemplo (además del de la barra del metro patrón) que refuerza la intuición kripkeana de que hay proposiciones a priori contingentemente verdaderas, $\mathrm{y}$, por tanto, que consiguen objetar el dogma kantiano según el cual necesidad y aprioridad son sinónimos intercambiables. El siguiente ejemplo se debe a Gareth Evans, ${ }^{59}$ y retomo la exposición de Papineau al respecto:

Supongamos que estamos discutiendo la historia de las invenciones

${ }^{57}$ Ibid., 501.

${ }^{58}$ Agustín Rayo, The construction of logical space (Oxford: Oxford University Press, 2015), 52.

${ }^{59}$ Gareth Evans, Ensayos filosóficos, trad. por Alejandro Tomasini Bassols (México: UNAM, Instituto de Investigaciones Filosóficas, 1996), 199-235. y estamos particularmente interesados en qué tipo de persona podría haber inventado la cremallera. Pero nos aburrimos de tener que decir "el inventor de la cremallera" todo el tiempo, de modo que adoptamos el nombre "Julius" para referirnos al inventor de la cremallera, quienquiera que pueda haber sido. Ahora, dada esta convención, el enunciado de que Julius inventó la cremallera (asumiendo que tuvo un único inventor) es ciertamente a priori. No necesitamos investigar el mundo para asegurarnos de que fue Julius quien inventó la cremallera. El enunciado de que Julius inventó la cremallera puede ser a priori, pero ciertamente no es necesario. Julius pudo haberse caído de cabeza cuando era pequeño y haber crecido demasiado estúpido para inventar la cremallera. O un desafortunado amorío podría haber hecho que se uniera a la Legión Extranjera francesa antes de haber hecho su innovación. El enunciado de que Julius inventó la cremallera bien podría haber sido falso si las cosas hubieran resultado ser distintas. De modo que este enunciado es tanto a priori como contingente. ${ }^{60}$

${ }^{60}$ David Papineau, Philosophical devices: Proofs, probabilities, possibilities, and sets (Oxford: Oxford University Press, 2012), 60-61. 